\title{
Characterization of shifts of koala (Phascolarctos cinereus) intestinal microbial communities associated with antibiotic treatment
}

\author{
Katherine E Dahlhausen ${ }^{\text {Corresp.. }}{ }^{1}$, Ladan Doroud ${ }^{2}$, Alana J Firl ${ }^{1}$, Adam Polkinghorne ${ }^{3}$, Jonathan A Eisen ${ }^{1}$ \\ ${ }^{1}$ Genome Center, University of California, Davis, Davis, CA, United States \\ 2 Department of Computer Science, University of California Davis, Davis, CA, United States \\ ${ }^{3}$ Centre for Animal Health Innovation, University of the Sunshine Coast, Sippy Downs, QLD, Australia \\ Corresponding Author: Katherine E Dahlhausen \\ Email address: katdah@ucdavis.edu
}

Koalas (Phascolarctos cinereus) are arboreal marsupials native to Australia that eat a specialized diet of almost exclusively eucalyptus leaves. Microbes in koala intestines are known to break down otherwise toxic compounds, such as tannins, in eucalyptus leaves. Infections by Chlamydia, obligate intracellular bacterial pathogens, are highly prevalent in koala populations. If animals with Chlamydia infections are received by wildlife hospitals, a range of antibiotics can be used to treat them. However, previous studies suggest that koalas can suffer adverse side effects during antibiotic treatment. This study aims to use $16 S$ rRNA gene sequences derived from koala feces to characterize the intestinal microbiome of koalas throughout antibiotic treatment and identify specific taxa associated with koala health after treatment. Although differences in the alpha diversity were observed in the intestinal flora between treated and untreated koalas and between koalas treated with different antibiotics, these differences were not statistically significant. The alpha diversity of koalas that lived through antibiotic treatment versus those did not was significantly greater, however. Beta diversity analysis largely confirmed the latter observation, revealing that the overall communities were different between koalas on antibiotics that died versus those that survived or never received antibiotics. Using both machine learning and OTU (operational taxonomic unit) co-occurrence network analyses, we found that OTUs that are very closely related to Lonepinella koalarum, a known tannin degrader found by culture-based methods to be present in koala intestines, was correlated with a koala's health status. This is the first study to characterize the time course of effects of antibiotics on koala intestinal microbiomes. Our results suggest it may be useful to pursue alternative treatments for Chlamydia infections without the use of antibiotics or the development of Chlamydia-specific antimicrobials that do not broadly affect microbial communities. 
1 Title: Characterization of shifts of koala (Phascolarctos cinereus) intestinal microbial

2 communities associated with antibiotic treatment

Authors: Katherine Dahlhausen ${ }^{1}$, Ladan Doroud ${ }^{2}$, Alana Firl ${ }^{1}$, Adam Polkinghorne ${ }^{3}$, Jonathan

5 A. Eisen $^{1}$

$7{ }^{1}$ Genome Center, University of California, Davis, Davis, California, USA

8 2Department of Computer Science, University of California Davis, Davis, CA, USA

$9{ }^{3}$ Centre for Animal Health Innovation, University of the Sunshine Coast, Sippy Downs, QLD,

10 Australia

\section{Abstract}

14 Koalas (Phascolarctos cinereus) are arboreal marsupials native to Australia that eat a specialized

15 diet of almost exclusively eucalyptus leaves. Microbes in koala intestines are known to break

16 down otherwise toxic compounds, such as tannins, in eucalyptus leaves. Infections by

17 Chlamydia, obligate intracellular bacterial pathogens, are highly prevalent in koala populations.

18 If animals with Chlamydia infections are received by wildlife hospitals, a range of antibiotics can

19 be used to treat them. However, previous studies suggest that koalas can suffer adverse side

20 effects during antibiotic treatment. This study aims to use $16 \mathrm{~S}$ rRNA gene sequences derived

21 from koala feces to characterize the intestinal microbiome of koalas throughout antibiotic

22 treatment and identify specific taxa associated with koala health after treatment. Although

23 differences in the alpha diversity were observed in the intestinal flora between treated and

24 untreated koalas and between koalas treated with different antibiotics, these differences were not

25 statistically significant. The alpha diversity of koalas that lived through antibiotic treatment

26 versus those did not was significantly greater, however. Beta diversity analysis largely confirmed

27 the latter observation, revealing that the overall communities were different between koalas on 
28 antibiotics that died versus those that survived or never received antibiotics. Using both machine

29 learning and OTU (operational taxonomic unit) co-occurrence network analyses, we found that

30 OTUs that are very closely related to Lonepinella koalarum, a known tannin degrader found by

31 culture-based methods to be present in koala intestines, was correlated with a koala's health

32 status. This is the first study to characterize the time course of effects of antibiotics on koala

33 intestinal microbiomes. Our results suggest it may be useful to pursue alternative treatments for

34 Chlamydia infections without the use of antibiotics or the development of Chlamydia-specific

35 antimicrobials that do not broadly affect microbial communities.

37 I. Background and Significance

38 The koala, Phascolarctos cinereus, is an arboreal marsupial native to Australia with multiple

39 unique aspects to its biology. Joeys (baby koalas) live in their mother's pouch, relying on koala

40 milk for nutrition for the first two months of life prior to switching to the consumption of pap for

41 up to another six months. Pap is fecal matter excreted by the mother, which is more concentrated

42 in nutrients and microbes than normal feces [1]. Pap consumption is an essential physiological

43 activity for joeys as they transition to the adult koala diet consisting almost exclusively of 44 eucalyptus leaves [1][2].

46 Eucalyptus leaves are high in tannins, soluble phenolic compounds that form complexes with 47 proteins and are resistant to degradation, rendering them toxic to many species that eat them [3].

48 It is thought that koalas rely on tannin-degrading bacteria that colonize the koala intestines once

49 a joey begins consuming pap from its mother [1][4]. Culture-based methods have revealed

50 Streptococcus sp. and Lonepinella koalarum as two known tannin degrading types of 
51 microorganisms that have been found in the gastrointestinal tract of koalas [5]. Tannin-

52 degrading bacteria are common amongst all animals with a high tannin diet, including koalas,

53 and allow these animals to survive off of tannin-rich diets [6][7].

55 One factor contributing to the dramatic decline in koala populations is infection by bacteria in 56 the Chlamydia genus, rates of which are as high as $100 \%$ in some koala populations [8].

57 Chlamydia are Gram negative intracellular bacterial pathogens, infecting a diversity of

58 eukaryotic hosts including mammals, birds, reptiles, fish, and amoeba [9]. Two species within

59 the genus Chlamydia are known to infect koalas, Chlamydia pecorum and Chlamydia

60 pneumoniae, with $C$. pecorum the most prevalent and pathogenic of the two in this host [10].

61

62 In koalas, Chlamydia infect the ocular site, urinary tract, and/or reproductive tract in both 63 chronic and acute states [10]. Wild koalas with symptoms of Chlamydia infections, such as 64 urinary incontinence or conjunctivitis, are routinely brought to wildlife hospitals to be tested and 65 treated [11][12]. Transmission of Chlamydiae between koalas can be sexually transmitted and 66 also through exposure to joeys when eating pap from an infected mother. [10] The treatment of

67 Chlamydia infections in koalas is controversial. Although different antibiotics are routinely 68 administered to koalas in care, several studies suggest antibiotic treatment has detrimental effects 69 on koalas such as a severe loss in body weight, severe dysbiosis, and even death [10][13][14].

70 Most notably, a few studies suggest that although effective, Chlamydia infection treatment with

71 the antibiotics chloramphenicol and enrofloxacin has adverse and even fatal side effects for

72 koalas $[13][15][16][17][18]$. A possibly related finding is that rats lacking tannin-degrading

73 bacteria while eating a tannin-enriched diet had similar symptoms (e.g. decreased food intake) to 
74 those reported for koalas on antibiotic treatment [6]. A study of antibiotic sensitivity of two

75 Lonepinella koalarum strains, known tannin-degraders, indicated sensitivity to chloramphenicol,

76 the antibiotic that all antibiotic-treated koalas in this study were administered [19] .

78 Despite the putative importance of koalas' intestinal microbes to their life biology, only two

79 culture-independent studies on the koala intestinal microbial community (microbiome) have been

80 published, neither in the context of the impacts of antibiotics [20][21]. In the current study, we

81 hypothesized that adverse side effects of antibiotics administered to koalas with Chlamydia

82 infections are related to disturbance in the microbial communities present in the koala

83 gastrointestinal tract. To test this hypothesis, we characterized over time the microbiome of

84 koalas that either were treated or not treated with antibiotics. We then analyzed these

85 microbiomes to examine how diversity patterns, individual taxa, and potential tannin degraders

86 varied with respect to koala survival and antibiotic treatment.

\section{II. Materials and Methods}

89 This study was conducted in close collaboration with the Australia Zoo Wildlife Hospital 90 (Queensland, Australia) and the Port Macquarie Koala Hospital (New South Wales, Australia).

91 Sample collection from the Australia Zoo Wildlife Hospital and Port Macquarie Koala Hospital 92 qualified for exemption from approval by the University of the Sunshine Coast Ethics 93 Committee. Furthermore, appropriate permissions for transportation and use of samples for 94 educational purposes was confirmed for export from the Australian Government and import from 95 the United States Fish and Wildlife Service. 


\section{Sample Collection}

98 Samples were collected from koalas admitted to the Australia Zoo Wildlife Hospital in Beerwah,

99 Queensland, Australia and the Port Macquarie Koala Hospital, Port Macquarie, New South

100 Wales, Australia. Koalas were lodged in outdoor, single-occupancy enclosures. They were fed a

101 variety of fresh eucalyptus leaves that were collected daily from local forests. Standard disease

102 transmission prevention procedures were used at each hospital. Only individuals that were

103 sampled for at least 21 days were included in this study because we thought that given koalas'

104 slow metabolisms and digestive mobility we would still be able to capture immediate changes to

105 the intestinal microbiome in this 3-week period. However, this resulted in a limited number of

106 control koalas because those that were not treated with antibiotics were released or died sooner

107 than our 21-day cut off.

108 Seven koalas from the Australia Zoo Wildlife Hospital were included, six of which received the

109 antibiotic chloramphenicol for the treatment of Chlamydia and one that did not receive any

110 antibiotic treatment. Treatment involved a single, daily treatment of $60 \mathrm{mg} / \mathrm{kg}$ for varying

111 durations; $\mathrm{mL}$ of the dosage is provided in Table 1. Four koalas were sampled from the Port

112 Macquarie Koala Hospital, three of which were administered a combination of chloramphenicol

113 and enrofloxacin antibiotics for the treatment of Chlamydia and one that did not receive any

114 antibiotic treatment. Treatment involved a single, daily treatment of $10 \mathrm{mg} / \mathrm{kg}$ of enrofloxacin for

1152 weeks, followed by a single, daily treatment of $60 \mathrm{mg} / \mathrm{kg}$ of chloramphenicol for varying

116 durations (Table 1).

117

118 Two animals from Australia Zoo Wildlife Hospital (antibiotic-treated) and one Port Macquarie

119 Koala Hospital animal (also antibiotic-treated), were euthanized during the course of this study. 
120 These animals exhibited too poor of a health status that they were determined to be unfit for

121 survival by their respective veterinarians.

122

123 A total of 141 fecal samples were collected for analysis in this study from the eleven animals

124 receiving care. These samples were composed of fresh fecal pellets $(<15$ minutes since

125 excretion) collected every three days, beginning on the day each koala was admitted (i.e.

126 immediately prior to first dosage of antibiotics) through to the day each koala was either released

127 back to the wild or deceased. Fecal material was collected from the floor or branch with gloved

128 hands and sealed in a small, sterile plastic bag. Samples were transported to a lab at the

129 University of the Sunshine Coast on ice $(<1 \mathrm{hr})$ from the zoo, and stored at $-80{ }^{\circ} \mathrm{C}$ (Table 1$)$.

131 Additionally, 17 built environment samples were collected from individuals' enclosures.

132 Samples were collected by a 10-second dry swabbing of floors and branches with a gloved hand,

133 and sealed in a small, sterile plastic bag. Leaf samples were collected as well by removing a full

134 leaves from the branches provided for each enclosure and sealing them in a small plastic bag.

135 Samples were transported to a lab at the University of the Sunshine Coast on ice $(<1 \mathrm{hr})$ from the

136 zoo, and stored at $-80{ }^{\circ} \mathrm{C}$. These samples are included in Figure 1, but were otherwise not

137 included in this report.

\section{DNA Extraction}

140 Based on examination of previous work, we concluded that fecal pellets were the best possible

141 proxy for the overall intestinal microbial community without the use of invasive collection

142 techniques [21]. To collect fecal material from the inside of the fecal pellet, the pellet was placed 
143 partway into a sterile $2 \mathrm{ml}$ Eppendorf tube, the pellet was broken in half, and the innermost

144 material from the middle of the pellet was removed with clean, sterile forceps. For each sample,

145 approximately $150 \mathrm{mg}$ of the fecal pellet was transferred with sterile forceps into a new sterile 2

$146 \mathrm{ml}$ Eppendorf tube. DNA was then extracted from this inner-pellet material using the Qiagen

147 QIAamp Fast DNA Stool Mini Kit (following the manufacturer's protocol). DNA was eluted in

148 a final volume of $100 \mu 1$ in $1.5 \mathrm{ml}$ LoBind Eppendorf tubes and stored at $-80{ }^{\circ} \mathrm{C}$.

150 To confirm that there was bacterial DNA in the samples, we first tested for PCR amplification of

151 16S rRNA genes using full-length eubacterial 16S rRNA gene PCR primers, 27F (5'-

152 AGAGTTTGATCMTGGCTCAG-3') and 1492R (5'-TCNGGYTACCTTGTTACGACTT-3').

153 Amplifications were carried out in an Eppendorf Mastercycler® PCR Cycler in $50 \mu 1$ reactions

154 containing $5 \mu \mathrm{l}$ of the eluted DNA from each samples' extraction, $18 \mu$ sterile Mill-Q® $\mathrm{H}_{2} 0,25$

$155 \mu \mathrm{l}$ MyTaq $^{\mathrm{TM}}$ HS Mix 2x (Bioline), and $1 \mu \mathrm{l}$ of $0.2 \mu \mathrm{M}$ concentration of each primer $27 \mathrm{~F}$ and

156 1492R (Sigma-Aldrich). The cycling conditions were: (1) $95^{\circ} \mathrm{C}$ for 10 minutes, (2) 35 cycles of

15745 seconds at $95{ }^{\circ} \mathrm{C}, 1$ minute at $50{ }^{\circ} \mathrm{C}$, and 2 minutes 20 seconds at $72{ }^{\circ} \mathrm{C}$, (3) a final incubation

158 at $72{ }^{\circ} \mathrm{C}$ for 7 minutes and (4) holding at $4{ }^{\circ} \mathrm{C}$ upon completion. A negative control was included

159 in every reaction which replaced the $5 \mu \mathrm{l}$ of DNA with $5 \mu$ of sterile Mill-Q ${ }^{\circledR} \mathrm{H}_{2} 0$.

160 The PCR fragments were visualized by 45 minutes of $120 \mathrm{~V}$ electrophoresis on a $1 \%$ agarose gel

161 stained with ethidium bromide. All samples showed a prominent band at the expected length $(\sim$

1621465 base pairs) for bacterial 16S rRNA genes; no bands were visible with the negative control

163 of replacing the $5 \mu \mathrm{l}$ of DNA with $5 \mu \mathrm{l}$ of $\mathrm{H}_{2} \mathrm{O}$.

164

165 Sequencing 
166 PCR amplification of the V4 region of the 16S rRNA gene was performed using primers $515 \mathrm{~F}$

167 and 806R, as recommended by Caporaso et al. and modified by the addition of a custom barcode 168 system described previously by Lang, et al. [22][23]. In addition to primers, Invitrogen Platinum

169 SuperMix was also used to perform PCR on $5 \mathrm{ng}$ of DNA for each sample following previously

170 established protocols [23]. PCR cycling conditions were (1) $95{ }^{\circ} \mathrm{C}$ for 2 minutes, (2) 30 cycles of $17195{ }^{\circ} \mathrm{C}$ for 30 seconds, $55^{\circ} \mathrm{C}$ for 30 seconds, $72{ }^{\circ} \mathrm{C}$ for 1 minute, and (3) $72{ }^{\circ} \mathrm{C}$ for 3 minutes.

172 PCR cleanup and normalization was performed according the manufacturer protocol for the

173 Invitrogen 96 well SequalPrep Normalization Plate. The resulting DNA elution of all pooled

174 samples was further purified and concentrated according to the manufacturer protocol for the

175 NucleoSpin ${ }^{\circledR}$ Gel and PCR Clean-up Kit (Macherey-Nagel). All DNA was quantified with

176 Qubit® dsDNA HS Assay Kit (Thermo Fisher Scientific). Purified DNA of a final concentration

177 of $34.1 \mathrm{ng} / \mu \mathrm{l}$ was submitted for sequencing at the UC Davis Genome DNA Technologies Core

178 in a multiplexed Illumina MiSeq lane. We generated 8,889,513 $250 \mathrm{bp}$ pairs of raw reads and

179 7,020,375 253 bp merged reads, using a custom script (available in Github repository

180 https:/github.com/gjospin/scripts/blob/master/Demul_trim_prep.pl) to assign each pair of reads

181 from the custom dual barcode system to individual samples. All subsequent analysis of 182 sequences was done on the merged reads.

183

\section{Data Analysis}

185 Sequences were analyzed using the QIIME (Quantitative Insights Into Microbial Ecology) version 1.9.1 workflow [24]. Quality filtering and chimera removal were performed on the

187 sequencing reads before downstream analysis. Operational taxonomic units (OTUs) were picked 188 at $97 \%$ similarity with QIIME's script (pick_otus_through_otu_table.py) and clustered using the 
189 UCLUST algorithm [25]. Taxonomy was assigned using the taxonomy assignment script in

190 QIIME (assign_taxonomy.py) with the open reference database, BLAST and reads that were not

191 assigned a taxonomy via that approach were assigned using the RDP Classifier [26][27]. OTUs

192 with five or fewer sequence representatives were filtered out. Additionally, the data was rarefied

193 to 5,000 reads per sample. OTUs that did not have a taxonomic assignment at the 'order' level

194 were filtered out.

195

196 Analysis was performed on samples from 11 individual koalas, nine of which had been treated

197 with antibiotics and two that had not. Samples were collected every three to four days from the

198 day the koala was first administered antibiotics until they were released or deceased; there were

199 different numbers of samples collected from individual koalas because individual treatment time

200 was varied. To normalize the analysis, only seven samples were used from each individual: the

201 first sample, the last sample, and five samples that were selected to provide roughly equal

202 chronological spacing from the treatment period. To ensure we only included OTUs capable of

203 conveying group-level effects, we filtered the relative OTUs abundance table to only include

204 OTUs that are present in two or more samples. All OTUs that did not satisfy this threshold were

205 grouped together (sum of their abundances per sample) so that the underlying distribution of

206 OTU abundances across samples did not change. This resulted in 1,511 OTUs across 77 samples.

207 All negative controls for the DNA extraction kit, PCR, and sequencing were below the minimum

2085,000 counts per sample that we set (see above) and thus were excluded from downstream

209 analysis. Koalas R, P, and J were treated with a second antibiotic in addition to the same

210 antibiotic as koalas A, B, D, E, F and G. However, calculations using the software R [28] 
211 determined no statistical difference existed between these two treatment groups (see Results) so

212 they were pooled into a single antibiotic group.

213

\section{Identifying Predictive OTUs of Fate: Random Forest Analysis}

215 To test whether a given set of OTUs was predictive of koala fate, and thus identify correlations

216 between survival and intestinal microbiome composition, we applied the Random Forest model

217 (available in Python's scikit-learn package [29]), a supervised machine-learning technique, to our

218 dataset. In our model, we assigned OTUs as the features and identified the set of OTUs that can

219 more accurately predict the fate (i.e. lived or died after treatment) of each individual. Using the

220 Random Forest model, we examined the microbiome of all of the individuals in the antibiotic

221 treatment group and determined the set of OTUs that create the highest distinction among the 222 individuals that survived and the individuals that died.

223

224 The goal of the Random Forest classifier is to learn dependencies and complex relationships 225 (both linear and nonlinear) among the features (here, relative OTU abundances) and find a set of

226 features that are the most discriminatory among the groups and can improve the predictive 227 accuracy of the model. It is important to note that the set of OTUs found in Random Forest 228 model can be a combination of both abundant and relatively rare OTUs across samples.

230 Finally, there is an importance score assigned to each OTU that shows how predictive that OTU

231 is in classifying the output. We measured the success of the Random Forest model by k-Fold 232 cross-validation; this includes training the model on a subset of samples and then using the 233 patterns learned to classify the remaining samples that were not used in the training step. 


\section{Diversity Analysis}

236 For the purpose of tracking antibiotic effect on the intestinal microbiome diversity, the mean

237 alpha diversity of each sample was measured by the Shannon Diversity Index using the vegan

238 package in $\mathrm{R}$ to measure variation within samples (i.e. alpha diversity) [28][30] (Figure 2).

239 Statistical significance of the differences in alpha diversity between groups was calculated using

240 a Welch two-sample t-test in R. To determine variations in microbial communities between

241 samples, principal-coordinate analysis (PCoA) plots were generated for weighted UniFrac,

242 unweighted UniFrac, and Bray-Curtis distances using QIIME software [31]. Statistical

243 significance of the differences between groups was calculated by either PERMANOVA or

244 ANOSIM with 9999 permutations in QIIME using the compare_categories.py script [24].

\section{OTU Co-Occurrence Network Analysis}

247 We investigated the potential interactions among various microbial taxa using network analysis.

248 It has been shown that studying OTU co-occurrences patterns using network analysis provide

249 insight into dynamics of complex microbial systems [32][33][34]. Here, we built a network of 250 the OTUs based on the presence/absence patterns of OTUs across koala samples using the first 251 and the last sample of individuals. Using our OTU table, each sample can be defined as a binary 252 vector of OTUs showing presence/absence of OTUs and thus each OTU can be defined as a 253 binary vector of that OTU presence/absence patterns across samples. To obtain co-occurrence 254 patterns among every pair of OTUs and thus building the OTU co-occurrences matrix, we used

255 the dot product of every two OTU vectors. The co-occurrence matrix was further used to build 256 the network. 
258 Each OTU represents a node in the network and there exists an edge between every two nodes if

259 they have co-occurred together. The edge weights are the dot product of each OTU vector pair 260 and are representative of how many times those two OTUs co-occurred together. We built a total 261 of three OTU co-occurrence networks for the following sample subsets: 1) The initial time points 262 for all individuals that were subjected to antibiotic treatment regardless of fate; 2) the final time 263 point for individuals who lived; and 3) the final time point for individuals who died. Note that 264 since we have smaller number of individuals in the deceased group, we expect to observe more 265 nodes in the network built from this group because it is more likely to see an OTU present in 266 three samples than it is to observe it in 6 individuals (case of released group) or 9 individuals 267 (network built based on the initial time point of all individuals). Once the networks were built, 268 we obtained clusters of OTUs that co-occurred together in each of these networks separately. We 269 looked at the differences among clusters of OTUs co-occurring in the initial sample and 270 compared that to OTU clusters that co-occurred in the last sampling. We then identified the 271 intersection of the co-occurring OTUs among these three networks.

\section{III. Results}

274 Using the QIIME (Quantitative Insights Into Microbial Ecology) workflow, taxonomic 275 assignments were made for 5934 OTUs (See Methods). After rarefaction to 5000 reads per 276 sample, the resulting OTU table consisted of 156 samples representative of various time-points

277 from 11 individual koalas. We removed OTUs that did not have any taxonomic assignment at the 278 'order' level. Finally, we selected seven samples from different time-points from each individual, 
279 including the sample collected the day each koala was to begin treatment and the last sample

280 collected before the individual was released or deceased for subsequent analysis.

281

282 To test for significant differences between metadata groups, Welch two-sample t-tests of the

283 mean alpha diversity were performed in R. Of these tests, we found no significant difference in

284 koala hospital location $(\mathrm{t}=0.544$, $\mathrm{df}=77.0, \mathrm{p}=0.587)$, koala sex $(\mathrm{t}=1.81, \mathrm{df}=86.2, \mathrm{p}=0.073)$,

285 antibiotic treatment regime $(\mathrm{t}=1.84, \mathrm{df}=52.9, \mathrm{p}=0.071)$, or whether the koala was administered

286 antibiotics $(\mathrm{t}=1.1413, \mathrm{df}=52.692, \mathrm{p}$-value $=0.2589)$. However, we did find whether a koala

287 lived through treatment $(\mathrm{t}=2.9239, \mathrm{df}=38.43, \mathrm{p}=0.005768)$ to be significant.

288

289 Diversity Analysis

290 Diversity analysis was performed on samples all time points for every koala as outlined in

291 Supplementary Table 1, with outliers removed where applicable, unless otherwise stated.

292 Alpha Diversity: Antibiotic vs. No Antibiotic. The average alpha diversity for each sample, as

293 calculated by the Shannon Diversity index, was not significantly different between samples from

294 koalas treated with antibiotics compared to samples from koalas that were not administered

295 antibiotics $(\mathrm{t}=1.1413, \mathrm{df}=52.692, \mathrm{p}=0.2589)$.

296 Alpha Diversity: Antibiotic and Fate. The average alpha diversity for samples from koalas that

297 lived through antibiotic treatment was found to be significantly greater than the average alpha

298 diversity for samples from koalas that died over the course of their admission, which included

299 antibiotic treatment $(\mathrm{t}=2.9239, \mathrm{df}=38.43, \mathrm{p}=0.005768)$ (Figure 2$)$.

300 Beta Diversity: Lived vs. Died. PCoA plots including all of the samples collected were

301 generated for three different distance-based calculation methods: Bray-Curtis, weighted Unifrac, 
302 and unweighted Unifrac (Figure 1). We found statistically significant differences in average

303 diversity between samples from antibiotic-treated koalas that lived to samples from antibiotic-

304 treated koalas that died for two of the three ordination methods used: Bray Curtis $(\mathrm{p}=0.0017)$,

305 unweighted UniFrac $(\mathrm{p}=0.0105)$. Weighted UniFrac was found to be not significant $(\mathrm{p}=0.1507)$.

306 Beta Diversity: Lived vs Control. For samples from koalas on antibiotics that lived to samples

307 from koalas not on antibiotics, differences in average diversity was found to be statistically

308 significant for Bray Curtis $(\mathrm{p}=0.0253$ ), unweighted UniFrac $(\mathrm{p}=0.0002)$, but not significant for

309 weighted UniFrac $(\mathrm{p}=0.2537)$.

310 Beta Diversity: Died vs Control. The difference in average diversity between samples from

311 koalas on antibiotics that died to samples from koalas not on antibiotics was found to be

312 statistically significant for all three ordination methods used: Bray Curtis $(\mathrm{p}=0.0001)$,

313 unweighted UniFrac $(\mathrm{p}=0.0001)$, and weighted UniFrac $(\mathrm{p}=0.0001)$.

316 Random Forest Analysis

317 Using the Random Forest model with a 3-fold cross-validation, we determined that individuals in

318 the antibiotic treatment group were classified accurately into two groups, those that survived and

319 those that died, with an accuracy of 92 percent and a total of 516 predictive OTUs. The OTU that

320 was most predictive of fate was classified (using the QIIME taxonomy assignment script

321 (assign_taxonomy.py) [24]) as in the genus Lonepinella (sp. koalarum). The distribution of the

322 feature importance was scored with the gini impurity criterion (measures the randomness of false

323 label assignment). Using this distribution, we identified the most predictive top 20 percent OTUs

324 and their distribution across samples (Figure 3). 


\section{OTU Co-occurrence Network Analysis}

327 The resulting bacterial networks for co-occurring OTUs all had a density of one, meaning that 328 there existed an edge between every two nodes (see Methods). Because all three networks 329 (antibiotic treated koalas $(\mathrm{N}=9)$, koalas that lived $(\mathrm{N}=6)$, and koalas that died $(\mathrm{N}=3)$ ) had a 330 density of one, they were defined as cliques and therefore considered to be one cluster. In more

331 detail, the network that was built based on the initial time point from nine individuals resulted in 33234 nodes with 561 edges. The average weighted degree was 297. Similarly, for the network built 333 for the final time point for three individuals that were deceased, there were 100 nodes with 4950 334 edges with an average weighted degree of 297. For the network of six released individuals, there 335 were 55 nodes with 1485 edges and average weighted degree of 324. As a final step, we looked 336 at the intersection of co-occurring OTUs among the networks we built. 24 of the OTUs that were 337 co-occurring at the initial time point still existed in all of the samples in both deceased and 338 released individuals at the final time point. There were four OTUs we determined to be of most 339 interest, New.ReferenceOTU131, New.ReferenceOTU23, New.ReferenceOTU271 and 340 New.ReferenceOTU265, which existed in all samples at initial time point and existed in all 341 released samples. However, they were absent from several samples of koalas that died. We note, 342 the taxonomic identification suggests that New.ReferenceOTU265 is most closely related to 343 sequences that were annotated as being in the Lonepinella genus. For more information on the 344 OTUs that resulted from network analysis, see Supplementary Table 1. 345 
347 While antibiotics have many benefits, they also can cause a disturbance to the microbial

348 communities of the host. Koalas are frequently administered antibiotics for extremely prevalent

349 Chlamydia infections, but the effect of antibiotic treatments on their intestinal microbial

350 communities has not been investigated to date. We believe this is likely to be especially

351 important for koalas, particularly if, as discussed above, they are dependent on the microbes in

352 their intestinal to break down the toxic components of their eucalyptus diet. This study

353 characterized the intestinal microbial community of 11 koalas over time until they were released

354 or deceased, nine of which were administered antibiotics as treatment for Chlamydia infections.

355 Of the metadata collected (e.g. koala hospital, sex, etc), we found that whether an antibiotic-

356 treated koala lived or died to be the only parameter that was significantly correlated with patterns

357 in our analysis. Therefore, our paper focuses on the differences between koala treated with 358 antibiotics that lived and koalas treated with antibiotics that died.

360 Diversity Analysis

361 Comparisons between antibiotic treated and untreated individuals. Several studies have

362 shown antibiotic treatment can reduce alpha diversity of mammalian intestinal

363 microbiota.[35][36] However we did not observe a significant difference between samples from

364 koalas that were administered antibiotics compared to samples from koalas that were not 365 exposed to antibiotics (Figure 2). This result indicates that antibiotic treatment may have caused 366 compositional shifts within the intestinal microbiome, without affecting overall species diversity.

367 While the lack of significant changes in alpha diversity suggests that antibiotics have a subtle

368 effect on the microbiome, there are also other putative explanations for this lack of a difference

369 in alpha diversity in treated and untreated koalas, including: 1) low doses of antibiotics used 
370 here. There could be many explanations for this such as the antibiotic being metabolized quickly

371 and therefore not having as strong of an impact on intestinal microbiota, for example. 2) Possible

372 lack of an effect of these antibiotics on the microbial community composition in a way that

373 would be reflected alpha diversity analysis. 3) Possible effects of captivity (i.e. transitional

374 stress, environmental changes, etc) which may mask some of the effects of antibiotics (at least on

375 alpha diversity). We note, there are still differences in community composition in the antibiotic

376 treated vs. untreated samples - the lack of any significant difference is only seen regarding alpha

377 diversity.

378 We did not find a statistically significant difference in alpha diversity between the two antibiotic

379 treatment regimes used. It is not surprising that the two different antibiotic treatment methods

380 (Chloramphenicol vs. Enrofloxacin and Chloramphenicol) did not have statistically different

381 effects on the intestinal microbial community of koalas in part because both are broad-spectrum 382 antibiotics.

383

384 Comparisons between antibiotic treated koalas that lived and those that did not

385 We did find a statistically significant difference in the alpha diversity between samples from

386 koalas that were administered antibiotics that lived and samples from koalas that were

387 administered antibiotics and died. This result is consistent with our hypothesis that a higher

388 richness and evenness in the initial intestinal microbial community composition may be more

389 important to koala health during antibiotic treatment than the direct impact of the antibiotic

390 treatment itself. 
392 We examined the beta diversity of different treatment groups (antibiotics and lived, antibiotics

393 and died, no antibiotics) in a pairwise manner using three different metrics: Unweighted Unifrac,

394 weighted Unifrac, and Bray-Curtis. The Unweighted Unifrac metric measures the dissimilarities

395 in phylogenetic distances of OTUs that are present or absent from samples, while the weighted

396 UniFrac metric measures the dissimilarities in phylogenetic distances of OTUs that are present or

397 absent from samples weighted by the abundance of those OTUs. The Bray-Curtis metric takes

398 into account the abundance of present or absent OTUs independent of phylogeny. The

399 differences in microbial communities between samples from koalas on antibiotics that lived

400 versus samples from koalas on antibiotics that died were found to be statistically significant for

401 the Bray Curtis and unweighted Unifrac metrics but not for weighted Unifrac. We interpret these

402 results as showing that the there are differences in both the abundance of specific OTUs (as

403 measured by the Bray-Curtis metric) and phylogenetic diversity of OTUs (as measured by

404 unweighted Unifrac). We are not certain why the weighted Unifrac analysis did not show

405 significant differences but we note that others have reported seeing significant differences in

406 communities for Bray-Curtis and weighted UniFrac metrics but not for unweighted UniFrac

407 [37][38]. This may be related to the method of randomization used in the weighted UniFrac

408 calculation for which there is some debate [38][39].

409

410 We also compared the samples from koalas on antibiotics that lived and samples from koalas on

411 antibiotics that died to samples from koalas that were never administered antibiotics. We found

412 the difference in average diversity between samples from koalas on antibiotics that lived to

413 samples from koalas not on antibiotics to be significant for Bray Curtis and unweighted UniFrac

414 metrics, but not significant for weighted UniFrac. The difference in average diversity between 
415 samples from koalas on antibiotics that died to samples from koalas not on antibiotics was found

416 to be statistically significant for all three ordination methods used: Bray Curtis, unweighted

417 UniFrac, and weighted UniFrac. These results indicate that the structure of the overall

418 communities are different between koalas that died on antibiotic treatment compared to koalas

419 that survived antibiotic treatment or were in the control group. These beta diversity results are

420 consistent with the alpha diversity results discussed above. One possible explanation for this is

421 that the initial community composition and structure are important to surviving antibiotic

422 treatment. However, we are not able to rule out other possible explanations with the data

423 available at this time.

424

$425 \underline{\text { Random Forest and Co-occurrence Network Analysis }}$

426 To identify the most predictive OTUs correlated with whether or not a koala survived antibiotic

427 treatment, we performed a Random Forest analysis of OTU relative abundance and presence /

428 absence. The Random Forest analysis revealed that an OTU identified as Lonepinella koalarum

429 is the most predictive OTU of whether or not a koala lived or died following the administration

430 of antibiotics. Koalas that died after antibiotic treatment had much lower relative abundance

431 (sometimes even zero), of this L. koalarum OTU compared to koalas that survived antibiotic

432 treatment (Figure 3). We believe that this correlation of the abundance of L. koalarum in koala

433 intenstines with surviving antibiotic treatment is potentially a key finding, as L. koalarum is

434 known to be a tannin-degrading microbe in the koala intestinal [1]. Our finding that the set of

435 OTUs predictive of koala survival classified with an accuracy of $92 \%$ suggests there is a strong

436 correlation between intestinal microbiome composition and koala prognosis, which is consistent

437 with our initial hypothesis. 
439 In addition to the Random Forest analysis, an OTU co-occurrence network analysis also revealed

440 that L. koalarum was correlated with whether or not koalas lived after antibiotic treatment. $L$.

441 koalarum was one of only four OTUs that was present at the beginning and at the end of

442 antibiotic treatment in koalas that lived, but absent in at least one koala that died after antibiotic

443 treatment.

444 Another finding from our Random Forest analysis was that the OTU "Cyanobacteria_YS2" was 445 in the top 30 most predictive of whether or not a koala lived following antibiotic treatment. 446 Recently, it was shown that the group to which this OTU was assigned to is actually 447 Melainabacteria, a new phylum of non-photosynthetic Cyanobacteria that has been found in 448 numerous mammalian intestines [40][41].

450 Overall, the results of Random Forest and co-occurrence network analyses support our 451 hypothesis that the administration of antibiotics, regardless of the combination of the treatment, 452 was associated with a change in the presence and relative abundance of L. koalarum, a known 453 tannin-degrader. This is not only important for veterinarians to consider when administering 454 antibiotics to koalas, but also for the development of Chlamydia infection treatments that do not 455 impact this critical intestinal microbe.

457 Potential confounding variables that may have influenced our results are sample storage [42], 458 DNA extraction method [43], PCR [44][45], and sequencing [46]. Due to not having full medical 459 histories of the koalas in this study, it is also possible that unknown confounding variables (e.g. 460 immune status or prior environment exposures), rather than antibiotics, contributed to our results. 
461 Other environmental variables that may be confounding factors that we were unable to test for

462 include the effects of being handled by hospital staff, koala compliance with antibiotic treatment,

463 differences between wildlife hospitals' environments and procedures, and day-to-day care.

464

\section{Conclusions}

466 Our findings are consistent with numerous other papers that suggest antibiotic treatments can

467 cause a disturbance to intestinal microbial communities [47][48][49][50]. Such disturbances are

468 likely to be particularly important in species like koalas, where it is thought that the intestinal

469 microbial community may be required for survival (in this case, detoxifying their food). This is

470 in contrast to many other animals where the intestinal microbiome is important but not

471 necessarily essential for survival. In addition to showing differences in antibiotic treated and

472 untreated koalas, we also found differences in richness, evenness, and structure of intestinal

473 microbial communities in antibiotic treated koalas for those that survived versus those that did

474 not. In particular, we found that the relative abundance of some key OTUs are correlated with

475 survival. This is consistent with our hypothesis that koala survival after antibiotic treatment is

476 related to whether or not key OTUs persist after the antibiotic treatment. We believe this

477 suggests that there may be a need to develop alternative treatments for koala Chlamydia

478 infections without the use of antibiotics and/or supplementing the koala diet with probiotics

479 during antibiotic treatment. Furthermore, our conclusions may be transferable to other species

480 that consume high-tannin diets. Future studies about this topic could be more comprehensive in

481 scale (e.g., sample size) and depth (e.g., metagenome rather than 16s rRNA gene sequencing). It

482 would be valuable to track koalas over a longer period of time rather than just their time in

483 captivity. Furthermore, an important area to investigate is the role of pap consumption in the 
484 colonization and microbial community structure changes of koala intestines. Given our findings, 485 it would be particularly important to investigate the pap of antibiotic treated mother koalas and 486 the impacts this has on joey health.

487

488 Acknowledgments

489 We would also like to thank Cheyne Flanagan (Port Macquarie Koala Hospital), Rosie Booth 490 and Amber Gillet (Australia Zoo Wildlife Hospital) for coordinating and help with sampling the

491 koalas in the study. Special thanks to Alyce Taylor-Brown, Cassie Ettinger (ORCID 0000-0001492 7334-403X) and David Coil (ORCID 0000-0001-6049-8240) for their guidance and expertise 493 throughout different stages of this project. Thank you to Guillaume Jospin (ORCID 0000-0002494 8746-2632) for providing his bioinformatics skills. And lastly, thank you to the Bosward family 495 for their help with logistics.

\section{References}

499 [1] Osawa, R., Rainey, F., Fujisawa, T., Lang, E., Busse, H. J., Walsh, T. P., and

$500 \quad$ Stackebrandt, E., "Lonepinella koalarum gen. nov., sp. nov., a new tannin-protein

501 complex degrading bacterium,” Syst. Appl. Microbiol., vol. 18, no. 3, pp. 368-373, 1995.

502 [2] Cork, Steven J., Hume, I. D., and Dawson, T. J., "Digestion and metabolism of a natural

503 foliar diet (Eucalyptus punctata) by an arboreal marsupial, the koala (Phascolarctos

504 cinereus),”J. Comp. Physiol., vol. 153, no. 2, pp. 181-190, 1983.

505 [3] Aguilar, Cristóbal N., Rodriguez, Raúl, Gutiérrez-Sánchez, Gerardo, Augur, Christopher,

506 Favela-Torres, Ernesto, Prado-Barragan, Lilia A., Ram’lirez-Coronel, Ascensión, and 
507 Contreras-Esquivel, Juan C., "Microbial tannases: advances and perspectives," Appl.

$508 \quad$ Microbiol. Biotechnol., vol. 76, no. 1, pp. 47-59, 2007.

509 [4] Goel, Gunjan, Puniya, A. K., Aguilar, C. N., and Singh, Kishan, "Interaction of gut

510 microflora with tannins in feeds," Naturwissenschaften, vol. 92, no. 11, pp. 497-503,

5112005.

512 [5] Goel, Gunjan, Puniya, Anil Kumar, and Singh, Kishan, "Phenotypic characterization of 513 tannin--protein complex degrading bacteria from faeces of goat," Small Rumin. Res., vol.

$514 \quad 69$, no. 1, pp. 217-220, 2007.

515 [6] Kohl, Kevin D., Stengel, Ashley, and Dearing, M.Denise, "Inoculation of tannin-

516 degrading bacteria into novel hosts increases performance on tannin-rich diets," Environ.

$517 \quad$ Microbiol., 2015.

518 [7] Gasse, Michael, “The Tannase Gene: Metaphylogenomics, Global Distribution and

519 Presence in the Midgut Flora of the Forest Tent Caterpillar Malacosoma disstria

$520 \quad \mathrm{H}\{\ddot{\mathrm{u}}\}$ bner,” Concordia University, 2014.

521 [8] Kollipara, Avinash, Polkinghorne, Adam, Wan, Charles, Kanyoka, Pride, Hanger, Jon,

522 Loader, Joanne, Callaghan, John, Bell, Alicia, Ellis, William, Fitzgibbon, Sean, and

523 others, "Genetic diversity of Chlamydia pecorum strains in wild koala locations across

524 Australia and the implications for a recombinant C. pecorum major outer membrane

525 protein based vaccine," Vet. Microbiol., vol. 167, no. 3, pp. 513-522, 2013.

526 [9] Bachmann, Nathan L., Polkinghorne, Adam, and Timms, Peter, "Chlamydia genomics:

527 providing novel insights into chlamydial biology," Trends Microbiol., vol. 22, no. 8, pp.

$528 \quad 464-472,2014$.

529 [10] Polkinghorne, Adam, Hanger, Jon, and Timms, Peter, "Recent advances in understanding 
the biology, epidemiology and control of chlamydial infections in koalas," Vet. Microbiol., 2013.

532 [11] Gonzalez-Astudillo, Viviana, Allavena, Rachel, McKinnon, Allan, Larkin, Rebecca, and

533

534

535

536 Henning, Joerg, "Decline causes of Koalas in South East Queensland, Australia: a 17-year retrospective study of mortality and morbidity,"Sci. Rep., vol. 7, 2017.

[12] Griffith, Joanna E., Dhand, Navneet K., Krockenberger, Mark B., and Higgins, Damien P., "A retrospective study of admission trends of koalas to a rehabilitation facility over 30 years," J. Wildl. Dis., vol. 49, no. 1, pp. 18-28, 2013.

[13] Osawa, R. and Carrick, F. N., "Use of a dietary supplement in koalas during systemic antibiotic treatment of chlamydial infection," Aust. Vet. J., vol. 67, no. 8, pp. 305-307, 1990.

[14] Brown, Anthony Stephen, "Infertility in the female koala (Phascolarctos cinereus)," 1987.

[15] Black, L. A., Higgins, D. P., and Govendir, M., "In vitro activity of chloramphenicol, florfenicol and enrofloxacin against Chlamydia pecorum isolated from koalas (Phascolarctos cinereus)," Aust. Vet. J., vol. 93, no. 11, pp. 420-423, 2015.

[16] Black, L. A., Landersdorfer, C. B., Bulitta, J. B., Griffith, J. E., and Govendir, Merran, "Evaluation of enrofloxacin use in koalas (Phascolarctos cinereus) via population pharmacokinetics and Monte Carlo simulation," J. Vet. Pharmacol. Ther., vol. 37, no. 3, pp. 301-311, 2014.

[17] Govendir, M., Hanger, J., Loader, J. J., Kimble, B., Griffith, J. E., Black, L. A., Krockenberger, M. B., and Higgins, D. P., "Plasma concentrations of chloramphenicol after subcutaneous administration to koalas (Phascolarctos cinereus) with chlamydiosis," J. Vet. Pharmacol. Ther., vol. 35, no. 2, pp. 147-154, 2012. 
553 [18] Lawrence, Amba, Fraser, Tamieka, Gillett, Amber, Tyndall, Joel D. A., Timms, Peter,

554 Polkinghorne, Adam, and Huston, Wilhelmina M., "Chlamydia Serine Protease Inhibitor,

555 targeting HtrA, as a New Treatment for Koala Chlamydia infection,” Sci. Rep., vol. 6,

5562016.

557 [19] Osawa, Ro Stackebrandt, Erko S, “Lonepinella,” Bergey’s Man. Syst. Archaea Bact., 5582015.

559 [20] Alfano, Niccoló, Courtiol, Alexandre, Vielgrader, Hanna, Timms, Peter, Roca, Alfred L., and Greenwood, Alex D., "Variation in koala microbiomes within and between individuals: effect of body region and captivity status," Nat. Publ. Gr., 2015.

562

[21] Barker, Christopher J., Gillett, Amber, Polkinghorne, Adam, and Timms, Peter, "Investigation of the koala (Phascolarctos cinereus) hindgut microbiome via 16S pyrosequencing," Vet. Microbiol., vol. 167, pp. 554-564, 2013.

[22] Caporaso, J.Gregory, Lauber, Christian L., Walters, William A., Berg-Lyons, Donna, Huntley, James, Fierer, Noah, Owens, Sarah M., Betley, Jason, Fraser, Louise, Bauer, Markus, and others, "Ultra-high-throughput microbial community analysis on the Illumina HiSeq and MiSeq platforms," ISME J., vol. 6, no. 8, pp. 1621-1624, 2012.

[23] Lang, Jenna M., Eisen, Jonathan A., and Zivkovic, Angela M., "The microbes we eat: abundance and taxonomy of microbes consumed in a day's worth of meals for three diet types," PeerJ, vol. 2, p. e659, 2014.

[24] Caporaso, J.Gregory, Kuczynski, Justin, Stombaugh, Jesse, Bittinger, Kyle, Bushman, Frederic D., Costello, Elizabeth K., Fierer, Noah, Pena, Antonio Gonzalez, Goodrich, Julia K., Gordon, Jeffrey I., and others, "QIIME allows analysis of high-throughput community sequencing data," Nat. Methods, vol. 7, no. 5, pp. 335-336, 2010. 
576 [25] Edgar, Robert C., "Search and clustering orders of magnitude faster than BLAST,"

577 Bioinformatics, vol. 26, no. 19, pp. 2460-2461, 2010.

578 [26] Altschul, Stephen F., Gish, Warren, Miller, Webb, Myers, Eugene W., and Lipman, David J., "Basic local alignment search tool," J. Mol. Biol., vol. 215, no. 3, pp. 403-410, 1990.

580

[27] Wang, Qiong, Garrity, George M., Tiedje, James M., and Cole, James R., "Naive

Bayesian classifier for rapid assignment of rRNA sequences into the new bacterial taxonomy," Appl. Environ. Microbiol., vol. 73, no. 16, pp. 5261-5267, 2007.

[28] Team, R.Core and others, "R: A language and environment for statistical computing," 2013.

585

[29] Pedregosa, Fabian, Varoquaux, Gaël, Gramfort, Alexandre, Michel, Vincent, Thirion, Bertrand, Grisel, Olivier, Blondel, Mathieu, Prettenhofer, Peter, Weiss, Ron, Dubourg, Vincent, and others, "Scikit-learn: Machine learning in Python," J. Mach. Learn. Res., vol. 12, no. Oct, pp. 2825-2830, 2011.

[30] Oksanen, Jari, Blanchet, F.Guillaume, Kindt, Roeland, Legendre, Pierre, Minchin, Peter R., O’Hara, R. B., Simpson, Gavin L., Solymos, Peter, Stevens, M.Henry H., Wagner, Helene, and others, "Package 'vegan,"” Community Ecol. Packag. version, vol. 2, no. 9, 2013.

[31] Caporaso, J.Gregory, Lauber, Christian L., Walters, William A., Berg-Lyons, Donna, patterns of 16S rRNA diversity at a depth of millions of sequences per sample," Proc. Natl. Acad. Sci., vol. 108, no. Supplement 1, pp. 4516-4522, 2011.

[32] Barberán, Albert, Bates, Scott T., Casamayor, Emilio O., and Fierer, Noah, "Using network analysis to explore co-occurrence patterns in soil microbial communities," ISME 
J., vol. 6, no. 2, pp. 343-351, 2012.

600 [33] Faust, Karoline, Lima-Mendez, Gipsi, Lerat, Jean-Sébastien, Sathirapongsasuti, Jarupon F., Knight, Rob, Huttenhower, Curtis, Lenaerts, Tom, and Raes, Jeroen, “Cross-biome comparison of microbial association networks," Front. Microbiol., vol. 6, 2015.

[34] Faust, Karoline, Sathirapongsasuti, J.Fah, Izard, Jacques, Segata, Nicola, Gevers, Dirk, Raes, Jeroen, and Huttenhower, Curtis, "Microbial co-occurrence relationships in the human microbiome," PLoS Comput Biol, vol. 8, no. 7, p. e1002606, 2012. fluoroquinolones on human gut microbiota in relation to Clostridium difficile associated "Prolonged impact of antibiotics on intestinal microbial ecology and susceptibility to enteric Salmonella infection,” Infect. Immun., vol. 77, no. 7, pp. 2741-2753, 2009. diarrhea," PLoS One, vol. 9, no. 2, p. e89417, 2014. comparing microbial communities," Appl. Environ. Microbiol., vol. 71, no. 12, pp. 8228$8235,2005$.

617 [38] Lozupone, Catherine A. and Knight, Rob, "The unifrac significance test is sensitive to tree topology," BMC Bioinformatics, vol. 16, no. 1, p. 211, 2015.

619 [39] Long, Jeffrey R., Pittet, Vanessa, Trost, Brett, Yan, Qingxiang, Vickers, David, Haakensen, Monique, and Kusalik, Anthony, "Equivalent input produces different output in the UniFrac significance test," BMC Bioinformatics, vol. 15, no. 1, p. 278, 2014. 
622 [40] Soo, Rochelle M., Skennerton, Connor T., Sekiguchi, Yuji, Imelfort, Michael, Paech,

623

624

625

626

628

629

630

631

632

634

635

636

637

638

Samuel J., Dennis, Paul G., Steen, Jason A., Parks, Donovan H., Tyson, Gene W., and Hugenholtz, Philip, "An expanded genomic representation of the phylum Cyanobacteria," Genome Biol. Evol., vol. 6, no. 5, pp. 1031-1045, 2014.

[41] Di Rienzi, Sara C., Sharon, Itai, Wrighton, Kelly C., Koren, Omry, Hug, Laura A., Thomas, Brian C., Goodrich, Julia K., Bell, Jordana T., Spector, Timothy D., Banfield, Jillian F., and others, "The human gut and groundwater harbor non-photosynthetic bacteria belonging to a new candidate phylum sibling to Cyanobacteria," Elife, vol. 2, p. e01102, 2013.

[42] Lauber, Christian L., Zhou, Nicholas, Gordon, Jeffrey I., Knight, Rob, and Fierer, Noah, "Effect of storage conditions on the assessment of bacterial community structure in soil and human-associated samples," FEMS Microbiol. Lett., vol. 307, no. 1, pp. 80-86, 2010.

[43] Salter, Susannah J., Cox, Michael J., Turek, Elena M., Calus, Szymon T., Cookson, William O., Moffatt, Miriam F., Turner, Paul, Parkhill, Julian, Loman, Nicholas J., and Walker, Alan W., "Reagent and laboratory contamination can critically impact sequencebased microbiome analyses," BMC Biol., vol. 12, no. 1, p. 87, 2014.

[44] Brooks, J.Paul, Edwards, David J., Harwich, Michael D., Rivera, Maria C., Fettweis, Jennifer M., Serrano, Myrna G., Reris, Robert A., Sheth, Nihar U., Huang, Bernice, Girerd, Philippe, and others, "The truth about metagenomics: quantifying and counteracting bias in 16S rRNA studies," BMC Microbiol., vol. 15, no. 1, p. 66, 2015.

[45] Brown, Christopher T., Hug, Laura A., Thomas, Brian C., Sharon, Itai, Castelle, Cindy J., Singh, Andrea, Wilkins, Michael J., Wrighton, Kelly C., Williams, Kenneth H., and Banfield, Jillian F., "Unusual biology across a group comprising more than $15 \%$ of 
645 domain Bacteria," Nature, vol. 523, no. 7559, pp. 208-211, 2015.

646 [46] Schirmer, Melanie, Ijaz, Umer Z., D’Amore, Rosalinda, Hall, Neil, Sloan, William T., and 647 Quince, Christopher, "Insight into biases and sequencing errors for amplicon sequencing 648 with the Illumina MiSeq platform," Nucleic Acids Res., p. gku1341, 2015.

649 [47] Jakobsson, Hedvig E., Jernberg, Cecilia, Andersson, Anders F., Sjölund-Karlsson, Maria, 650 Jansson, Janet K., and Engstrand, Lars, "Short-term antibiotic treatment has differing 651 long-term impacts on the human throat and gut microbiome," PLoS One, vol. 5, no. 3, p.

652 e9836, 2010.

653 [48] Dethlefsen, Les, Huse, Sue, Sogin, Mitchell L., and Relman, David A., "The pervasive 654 effects of an antibiotic on the human gut microbiota, as revealed by deep 16S rRNA sequencing," PLoS biol, vol. 6, no. 11, p. e280, 2008.

656

657

[49] Jernberg, Cecilia, Löfmark, Sonja, Edlund, Charlotta, and Jansson, Janet K., "Long-term

658 ecological impacts of antibiotic administration on the human intestinal microbiota," ISME J., vol. 1, no. 1, pp. 56-66, 2007.

659 [50] Sullivan, Åsa, Edlund, Charlotta, and Nord, Carl Erik, "Effect of antimicrobial agents on 660 the ecological balance of human microflora," Lancet Infect. Dis., vol. 1, no. 2, pp. 101$661 \quad 114,2001$. 


\section{Figure 1}

Principle Coordinate Analysis ( $\mathrm{PCOA}$ ) of microbial communities found in koala fecal samples based on analysis of rRNA gene sequences.

PCoA plots were generated using the QIIME (Quantitative Insights Into Microbial Ecology) version 1.9.1 workflow on sequencing reads following quality filtering and chimera removal. PCOA plots are shown for multiple metrics to illustrate how beta diversity is related to abundance and phylogeny: unweighted Unifrac (A), weighted Unifrac (B), and Bray-Curtis (C). Each point represents a unique sample. Coloring indicates individual koalas, shape indicates type of treatment or sample (circles - antibiotic treated, triangles -no antibiotics, squares -samples from the built environment), fill indicates whether the koala lived or died.Numbers in legend indicate the number of samples for that koala.Colors were chosen based on Martin Krzywinski's Color Blindness Palette for improved viewing by those with color blindness. 

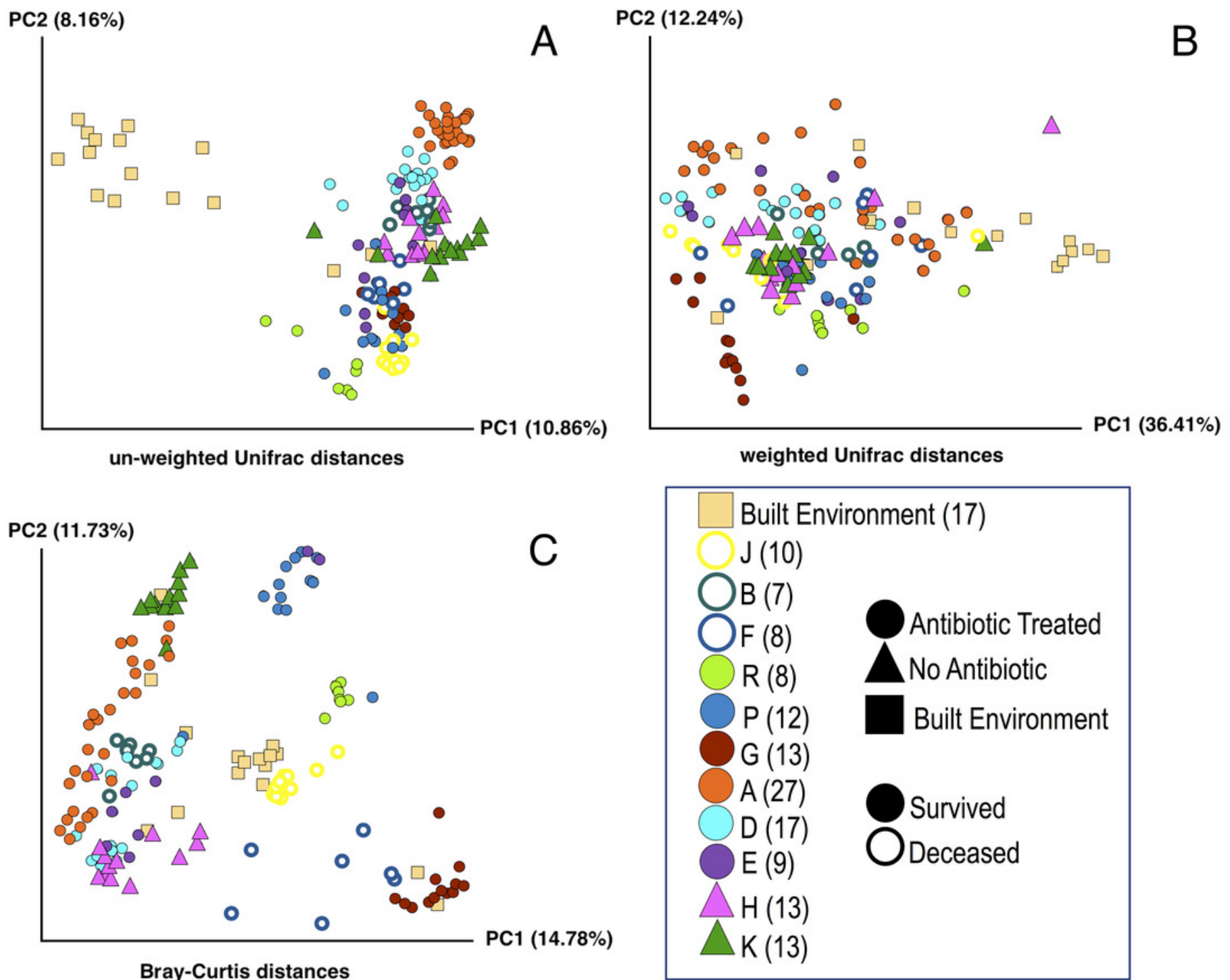

\begin{tabular}{|ll|}
\hline Built Environment (17) \\
$\mathrm{J}(10)$ & \\
$\mathrm{B}(7)$ & \\
$\mathrm{F}(8)$ & Antibiotic Treated \\
$\mathrm{R}(8)$ & $\mathbf{N}_{\text {No Antibiotic }}$ \\
$\mathrm{P}(12)$ & \\
$\mathrm{G}(13)$ & \\
$\mathrm{A}(27)$ & \\
$\mathrm{D}(17)$ & Suilt Environment \\
$\mathrm{E}(9)$ & ODeceased \\
$\mathrm{H}(13)$ & \\
$\mathrm{K}(13)$ & \\
\hline
\end{tabular}




\section{Figure 2}

Modified boxplots of alpha diversity (Shannon index) for microbial communities found in koala fecal samples based on analysis of rRNA gene sequences.

Data is presented for pools from different groups of koalas. The Shannon diversity index was calculated for each sample using $\mathrm{R}$ and averages for each group of interest were then calculated. The average alpha diversity for each sample was not significantly different between samples from koalas treated with antibiotics (t) test statistic) $=1.1413, \mathrm{df}$ (degrees of freedom $)=52.692, p$-value $=0.2589$ ). The average alpha diversity for samples from koalas that lived through antibiotic treatment was found to be significantly greater than the average alpha diversity for samples from koalas that died during their admission which included antibiotic treatment $(t=2.9239, d f=38.43, p=0.005768)$. Outliers (shown by open circles) represent values that are 1.5 times greater than the difference between the third and first quartiles of the data set. 


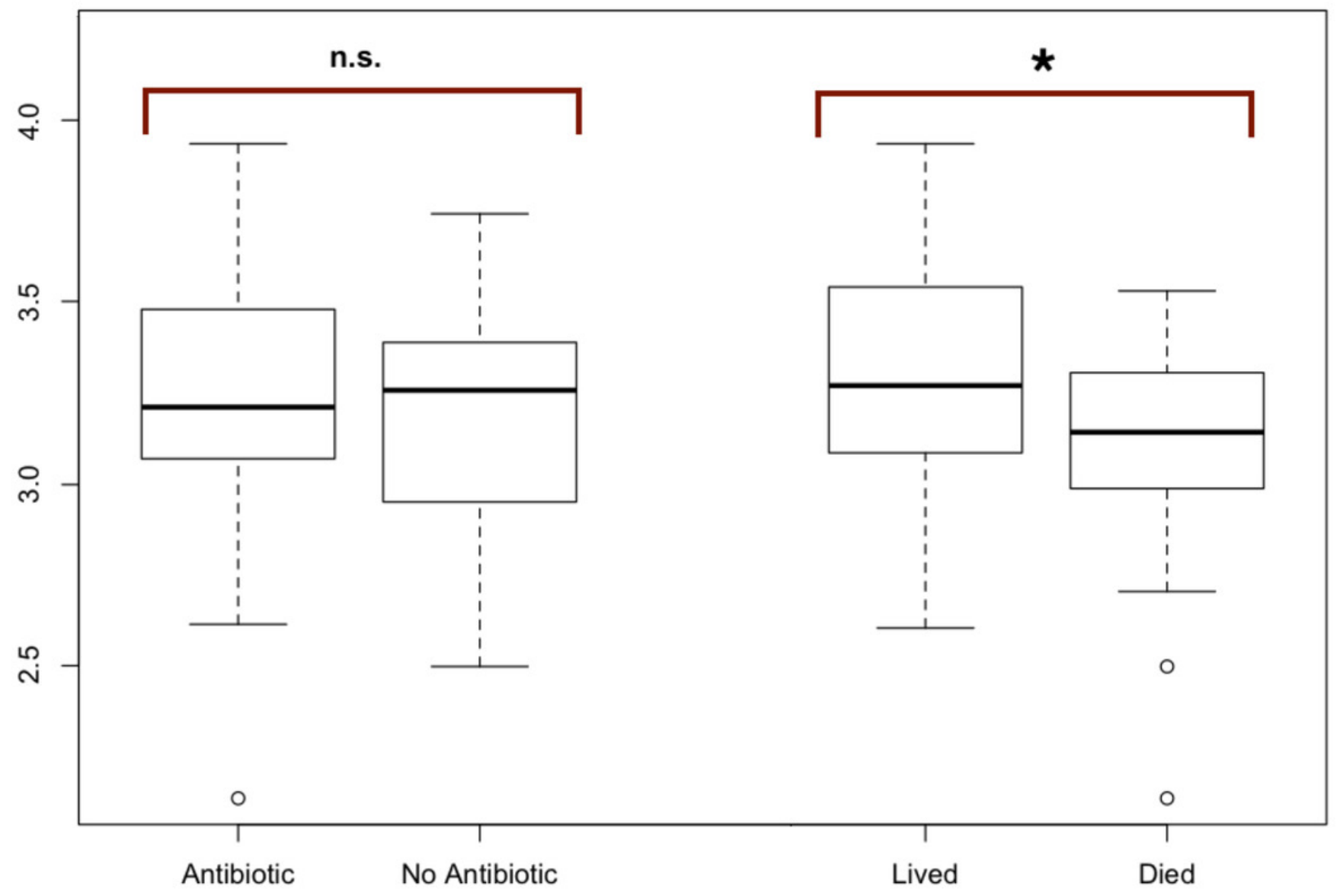




\section{Figure 3}

The distribution of the top $20 \%$ of feature-importance OTUs across individual samples as determined by Random Forest Analysis.

Individual samples on the $\mathrm{X}$ axis are organized by the individual koalas from which they came (represented by unique colors), and divided by 'Released' on the left and 'Deceased' on the right. The $Y$ axis represents the taxonomic assignment (see methods) for each of the top 20\% OTUs that were the most predictive of fate according to our Random Forest Analysis. The coloring of the text for the OTU names is used to highlight specific taxa of interest flagged by network analysis (see main text). Highlighted in red are OTUs identified as Lonepinella koalarum that the network analysis identified as the most predictive OTUs of fate. Highlighted in blue are OTUs identified as 'Cyanobacteria YS2', which were identified as being highly predictive of fate and are of interest because they are Melainabacteria. The density of each point in the heatmap is representative of the relative abundance of each OTU for each sample. 


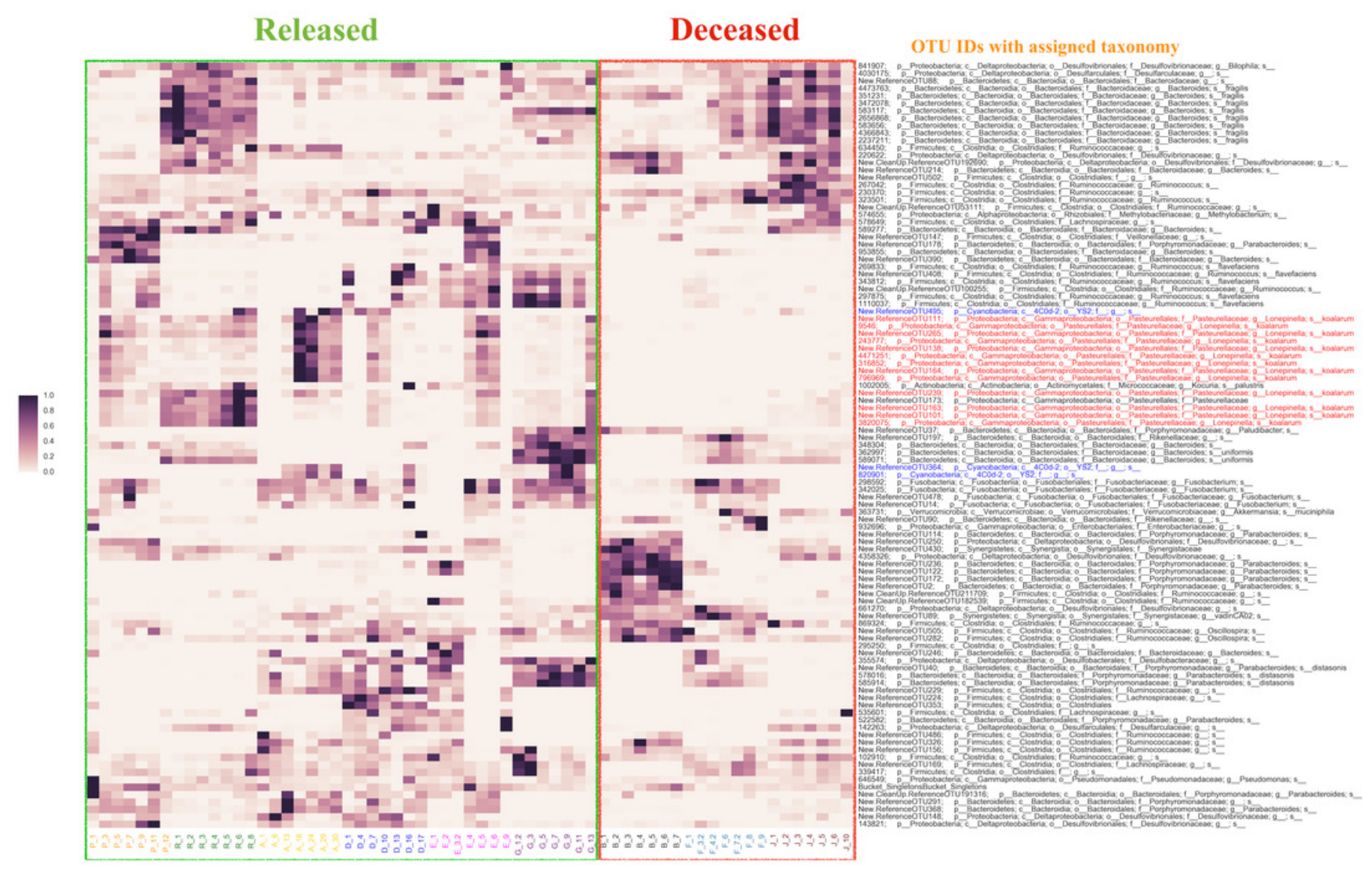




\section{Table $\mathbf{1}$ (on next page)}

Information on study cohort of koalas, treatments, and samples.

Table lists antibiotic used, dosage, fate and number of samples. Antibiotic treatment regime (type of antibiotic, dosage, and time course) and euthanasia decisions were determined by the assigned veterinarian for each koala. 


\begin{tabular}{|c|c|c|c|c|}
\hline Koala ID & Antibiotic Used & Daily Dose & $\begin{array}{l}\text { Lived or } \\
\text { Died }\end{array}$ & $\begin{array}{c}\text { No. Samples } \\
\text { Collected }\end{array}$ \\
\hline $\mathrm{A}$ & Chloramphenicol & $2.6 \mathrm{ml}$ Chloramphenicol & Lived & 30 \\
\hline $\mathrm{B}$ & Chloramphenicol & $2.0 \mathrm{ml}$ Chloramphenicol & Died & 7 \\
\hline $\mathrm{D}$ & Chloramphenicol & $2.5 \mathrm{ml}$ Chloramphenicol & Lived & 17 \\
\hline $\mathrm{E}$ & Chloramphenicol & $2.1 \mathrm{ml}$ Chloramphenicol & Lived & 9 \\
\hline $\mathrm{F}$ & Chloramphenicol & $2.3 \mathrm{ml}$ Chloramphenicol & Died & 9 \\
\hline $\mathrm{G}$ & Chloramphenicol & $1.9 \mathrm{ml}$ Chloramphenicol & Lived & 13 \\
\hline $\mathrm{H}$ & No Antibiotics & 0 & Lived & 13 \\
\hline $\mathrm{J}$ & $\begin{array}{l}\text { Chloramphenicol } \\
\text { and Enrofloxacin }\end{array}$ & $\begin{array}{c}1.4 \mathrm{ml} \text { Enrofloxacin, } \\
2.4 \mathrm{ml} \text { Chloramphenicol }\end{array}$ & Died & 10 \\
\hline $\mathrm{K}$ & No Antibiotics & 0 & Lived & 14 \\
\hline $\mathrm{P}$ & $\begin{array}{l}\text { Chloramphenicol } \\
\text { and Enrofloxacin }\end{array}$ & $\begin{array}{l}1.3 \mathrm{ml} \text { Enrofloxacin, } \\
2.6 \mathrm{ml} \text { Chloramphenicol }\end{array}$ & Lived & 12 \\
\hline $\mathrm{R}$ & $\begin{array}{l}\text { Chloramphenicol } \\
\text { and Enrofloxacin }\end{array}$ & $\begin{array}{c}1.1 \mathrm{ml} \text { Enrofloxacin, } \\
2.4 \mathrm{ml} \text { Chloramphenicol }\end{array}$ & Lived & 8 \\
\hline
\end{tabular}

1

2 